Díez, P., Parés, N. y Huerta, A., Accurate upper and lower error bounds by solving flux-free local problems in "stars", Revue européenne des éléments finis, Vol. 13, Issue 5-6-7/2004, pp. 497-507, 2004

\title{
Accurate upper and lower error bounds by solving flux-free local problems in "stars"
}

\author{
Pedro Díez - Núria Parés - Antonio Huerta \\ Universitat Politècnica de Catalunya \\ Departament de Matemàtica Aplicada III \\ E.T.S. de Ingenieros de Caminos, Canales y Puertos \\ E.U. de Ingenieros Técnicos Industriales de Barcelona \\ E-08034 Barcelona, Spain \\ pedro.diez@upc.es
}

\begin{abstract}
Two implicit residual type estimators yielding upper bounds of the error are presented which do not require flux equilibration. One of them is based on the ideas introduced in [MAC 00, CAR 99, MOR 03, PRU 02]. The new approach introduced here is based on using the estimated error function rather than the estimated error norms. Once the upper bounds are computed, also lower bounds for the error are obtained with little supplementary effort.

RÉSUMÉ. On présente deux estimateurs d'erreur qui ne nécessitent point d'équilibration locale de flux (contraintes) et qui fournissent des bornes supérieures de l'erreur. Le premier se base dans les idées introduites dans [MAC 00, CAR 99, MOR 03, PRU 02]. L'estimateur introduit ici suit la même philosophie mais se base dans l'estimation des fonctions d'erreur et pas seulement celle des normes. Une fois les estimateurs en borne supérieure calculés, des bornes inférieures peuvent être obtenues avec un petit effort suplémentaire.

KEYWORDS: error estimation, flux-free estimates, adaptivity, upper and lower bounds of the error. MOTS-CLÉS : estimation d'erreur, estimateurs sans flux, adaptivité, bornes supérieures (majorations) et inférieures (minorations) de l'erreur.
\end{abstract}




\section{Introduction}

The goal of the application of computational mechanics to engineering practice is often the evaluation of particular quantities of interest. These quantities are also denoted as engineering outputs of the problem. The goal of an adaptive procedure is to control efficiently the accuracy of these outputs, which represent the relevant engineering quantities. Recently, a number of researchers have addressed the problem of assessing and controlling the error of the numerical solution in these outputs of interest, instead of the classical energy norm error estimation.

It is worth noting that goal oriented error estimates have the attractive feature of being directly computable from energy estimates of the error. It suffices to apply them to the approximations of the primal and dual (with respect to the output) problems. In order to bound the error in the quantity of interest, simultaneous lower and upper bounds of the energy error are needed.

Many approaches for computing bounds on outputs, as [PAR 97] and [PRU 99], are based in this important property, thus, based on the obtention of accurate upper and lower bounds for the energy norm of the error.

In this work, a new approach to obtain simultaneous upper and lower bounds of the energy norm of the error is presented. The main advantage is that it does not require

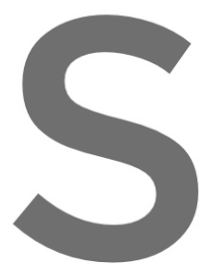
neither flux jur for assessing the compuring fluxes is very The approach described in this paper uses the solution associated with a fine truth
mesh as a reference solution. The reference mesh is much finer than the current orig-
nal mesh and therefore the reference solution is much closer to the unknown exact solution than the computed with the original mesh. Thus, a reference error $e_{\text {ref }}$ is

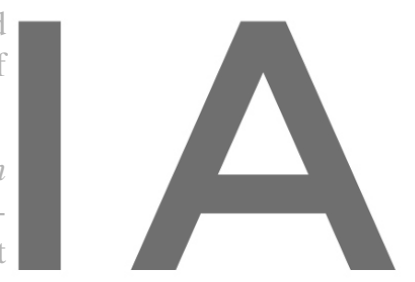

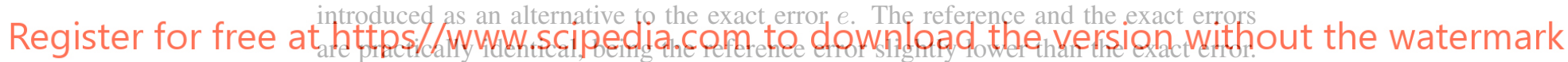

The error estimates introduced below are indeed upper and lower bounds of the error. However, the overestimation (upper bound) is only proved with respect to the reference error, not with respect to the exact error. It may happen that the error estimate ranges between the reference and the exact errors. In that case, paradoxically, the upper bound is underestimating the exact error. Some work has been devoted to obtain upper bounds with respect to the exact solution [BON 02]. These approaches are based on solving the local equations using equilibrium methods. Nevertheless, this problem arises only if the estimate is extremely sharp and the introduced overestimation with respect to the reference error is lower than the difference between the reference and the exact solution. In practice, this happens rarely.

\section{Model problem and error equations}

The model problem is stated as follows: find $u \in H_{\Gamma_{D}}^{1}(\Omega)$ such that

$$
a(u, v)=l(v), \quad \forall v \in H_{0}^{1}(\Omega),
$$


where $H_{\Gamma_{D}}^{1}(\Omega)$ and $H_{0}^{1}(\Omega)$ are the standard Sobolev spaces satisfying the Dirichlet and homogeneous boundary conditions respectively.

The finite element interpolation space and the finite element solution are denoted by $V^{h}$ and $u_{h}$ respectively, and the energy norm is denoted by $\|\cdot\|$. Let $x^{i}, i=$ $1 \ldots \mathrm{n}_{\text {node }}$ denote the nodes of the mesh and $\phi^{i} \in V^{h}$ the corresponding shape functions. The support of $\phi^{i}$ is denoted by $\omega^{i}$ and it is called the star centered in or associated with node $x^{i}$.

Note that the shape functions are a partition of unity,

$$
\sum_{i=1}^{\mathrm{n}_{\text {node }}} \phi^{i}(x)=1, \quad \forall x \in \Omega
$$

This essential property is used in the following to define residual estimators based in stars. Similar approaches have been used in references [BAB 78], [CAR 99] and [MOR 03].

We address the problem of assessing the error, $e:=u-u_{h} \in H_{0}^{1}(\Omega)$.

The global equation for the error is
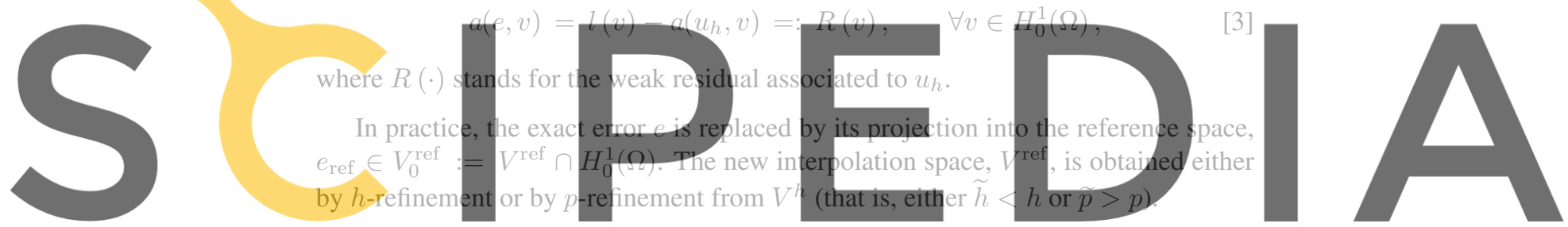

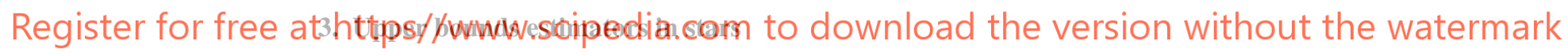

In this section, two estimates are presented, both providing an upper bound of the energy norm of the reference error.

The first strategy introduces a local approximation $e_{\mathrm{u}}^{\omega^{i}} \in V_{0, \omega^{i}}^{\text {ref }}$ of the contribution of the star $\omega^{i}$ to the reference error, where $V_{0, \omega^{i}}^{\text {ref }}$ denote the local restriction of the reference space to the $\operatorname{star} \omega^{i}$, that is, $V_{0, \omega^{i}}^{\text {ref }}:=V_{0}^{\text {ref }} \cap H^{1}\left(\omega^{i}\right)$. Then $e_{\mathrm{u}}^{\omega^{i}}$ is defined to be the solution of

$$
a_{\omega^{i}}\left(e_{\mathrm{u}}^{\omega^{i}}, v\right)=R\left(\phi^{i} v\right), \quad \forall v \in V_{0, \omega^{i}}^{\mathrm{ref}}
$$

where $a_{\omega^{i}}(\cdot, \cdot)$ is the restriction of the bilinear form $a(\cdot, \cdot)$ to the star $\omega^{i}$.

A global estimate of the error is defined adding the local contributions, that is

$$
e_{\mathrm{u}}:=\sum_{i=1}^{\mathrm{n}_{\text {node }}} e_{\mathrm{u}}^{\omega^{i}} .
$$


Note that $e_{\mathrm{u}}$ belongs to the "broken" space, that is, $e_{\mathrm{u}} \in V_{\mathrm{brok}}^{\mathrm{ref}}:=\bigoplus V_{0, \Omega_{k}}^{\mathrm{ref}}$, where $V_{0, \Omega_{k}}^{\text {ref }}$ is the restriction of the reference space to the element $\Omega_{k}$. In other words, the restriction of $e_{\mathrm{u}}$ to an element belongs to the reference space, and hence is continuous, but it is generally discontinuous across interelement boundaries.

The following theorem states that $e_{\mathrm{u}}$ defined in Eq. (5) behaves as an upper bound residual type error estimator based in a flux equilibration technique, see references [BAN 85] and [AIN 93].

Theorem 1. The estimate $e_{\mathrm{u}} \in V_{\mathrm{brok}}^{\mathrm{ref}}$ introduced in Eq. (5) verifies

$$
a\left(e_{\mathrm{u}}, v\right)=a\left(e_{\mathrm{ref}}, v\right), \quad \forall v \in V_{0}^{\mathrm{ref}} .
$$

Thus, the norm of $e_{\mathrm{u}}$ is an upper bound of the energy norm of the reference error, that

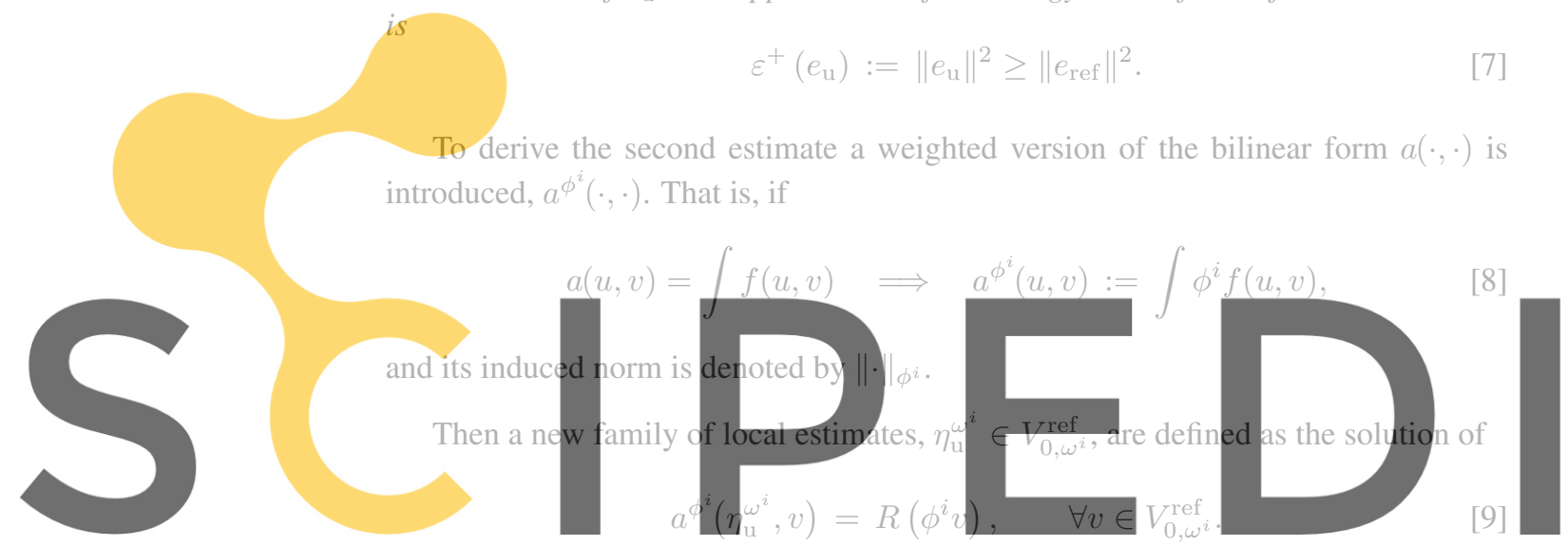

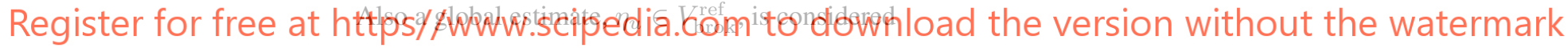

$$
\eta_{u}:=\sum_{i=1}^{n_{\text {node }}} \eta_{u}^{\omega^{i}} .
$$

Theorem 2. Let $\eta_{u}$ be the global estimate defined in Eq. (10). Then an upper bound is computed from $\eta_{u}$ using the weighted norms as

$$
\varepsilon^{+}\left(\eta_{u}\right):=\sum_{i=1}^{\mathrm{n}_{\text {node }}}\left\|\eta_{\mathrm{u}}^{\omega^{i}}\right\|_{\phi^{i}}^{2} \geq\left\|e_{\mathrm{ref}}\right\|^{2} .
$$

REMARK.- Note that upper bound estimates are obtained without any flux recovery or flux splitting technique. The effect of the flux jumps across each edge of the mesh is implicitly taken into account because the support of the local problems are the stars, which include the interelement edges. There is no need of computing and postprocessing the fluxes of the finite element solution, $u_{h}$, along the interelement edges. 


\section{Lower bound recovering}

The idea of obtaining a lower bound by a simple post-processing of the upper bound error estimate, is to construct continuous estimates from them. A simple averaging technique can be considered [DIE 03]. However, in this case, the particular form of the estimates $e_{\mathrm{u}}$ and $\eta_{u}$ can be taken into account in order to improve the lower bounds.

The idea is that the local estimates, $e_{\mathrm{u}}^{\omega^{i}}$ and $\eta_{\mathrm{u}}^{\omega^{i}}$, are discontinuous along the boundary of the star $\omega^{i}$. In order to enforce continuity it suffices to consider $\phi^{i} e_{\mathrm{u}}^{\omega^{i}}$ and $\phi^{i} \eta_{\mathrm{u}}^{\omega^{i}}$.

Therefore $e_{\mathrm{W}}$ and $\eta_{\mathrm{W}}$ given by

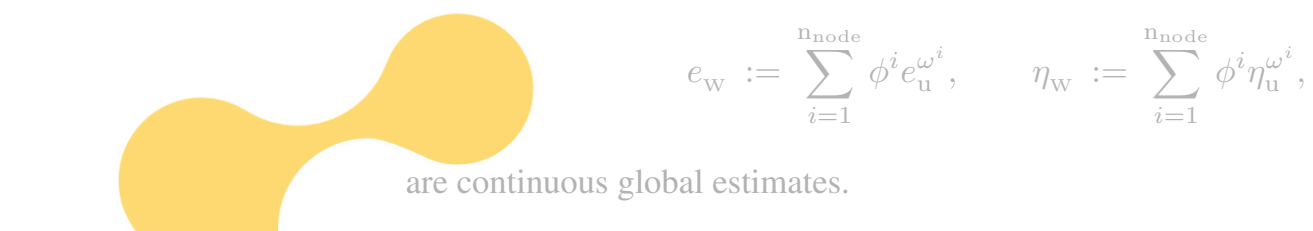

are continuous global estimates

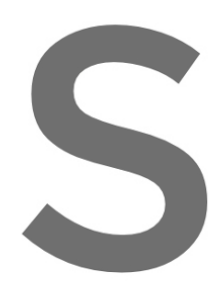

Theorem 3. Let $e_{u}^{\omega^{i}}, \eta_{u}^{\omega^{i}} \in V_{0, \omega^{i}}^{\mathrm{ref}}, i=1 \ldots \mathrm{n}_{\text {node }}$ be the estimates obtained solving

Eqs. (4) and (9) respectively. Then the continuous estimates $e_{\mathrm{W}}$ and $\eta_{\mathrm{w}}$ defined in Eq.

(12) yield lo
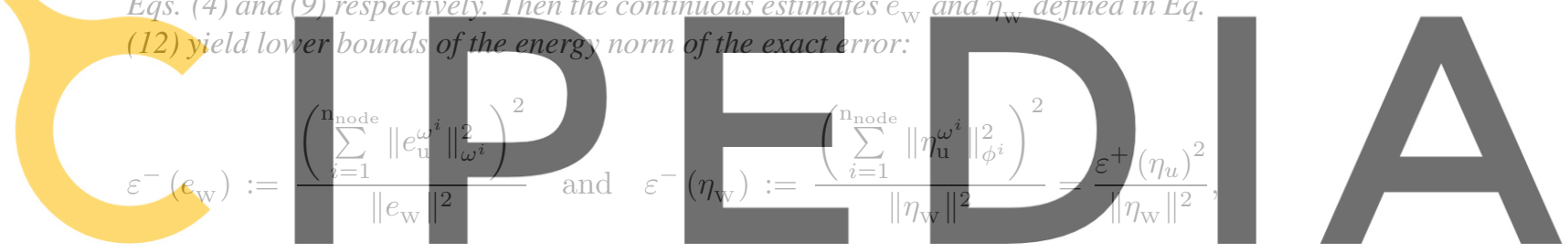

where $\|\cdot\|_{\omega^{i}}$ stands for the norm associated to the local bilinear form $a_{\omega^{i}}(\cdot, \cdot),\|v\|_{\omega^{i}}^{2}=$

$$
\varepsilon^{-}\left(e_{\mathrm{W}}\right) \leq\|e\|^{2} \quad \text { and } \quad \varepsilon^{-}\left(\eta_{\mathrm{W}}\right) \leq\|e\|^{2} .
$$

To improve this lower bounds, once the continuous estimate is computed, a global error assessment is introduced to take into account pollution errors [HUE 00]. Following this strategy, two new estimates, $e_{\mathrm{w}}^{\mathrm{G}}$ and $\eta_{\mathrm{w}}^{\mathrm{G}}$, are obtained using $e_{\mathrm{w}}$ and $\eta_{\mathrm{w}}$. Both $e_{\mathrm{w}}^{\mathrm{G}}$ and $\eta_{\mathrm{w}}^{\mathrm{G}}$ are continuous and provide new lower bounds for the error. The lower bounds provided by $e_{\mathrm{w}}^{\mathrm{G}}$ and $\eta_{\mathrm{W}}^{\mathrm{G}}$ are sharper than the lower bounds provided by $e_{\mathrm{w}}$ and $\eta_{\mathrm{w}}$.

\section{Numerical examples}

In this section, the behavior of the estimates introduced in the previous sections is analyzed using two numerical examples: one thermic (scalar unknown) and one mechanical (vectorial unknown). 
The quality of the error estimates is measured with the index $\rho$, defined by

$$
\rho:=\frac{\text { estimated error }}{\text { reference error }}-1,
$$

that is, $\rho$ is the effectivity index minus one. The quality of the estimates is given by the absolute value of $\rho$ and the sign of $\rho$ indicates if the estimate is an upper (positive $\rho$ ) or a lower bound (negative $\rho$ ).

In the examples bilinear finite elements are used $(p=1)$, and the reference space $V^{\text {ref }}$ is obtained refining the initial mesh ( $h$-refinement).

Four error estimates are considered: two upper-bound estimates, $e_{\mathrm{u}}$ and $\eta_{u}$, and two continuous estimates $e_{\mathrm{W}}^{\mathrm{G}}$ and $\eta_{\mathrm{W}}^{\mathrm{G}}$, which provide lower bounds for the error.

\subsection{Example 1: thermal problem}

The Poisson equation is solved in a squared domain, $\Omega$, with boundary conditions Dirichlet homogeneous on $\Gamma_{D}:=\{(x, 0) ; 0 \leq x \leq 1\}$ and Neumann homogeneous elsewhere on $\partial \Omega$. The source term $s$ is taken such that the exact solution has the following analytical expression (Figure (1)
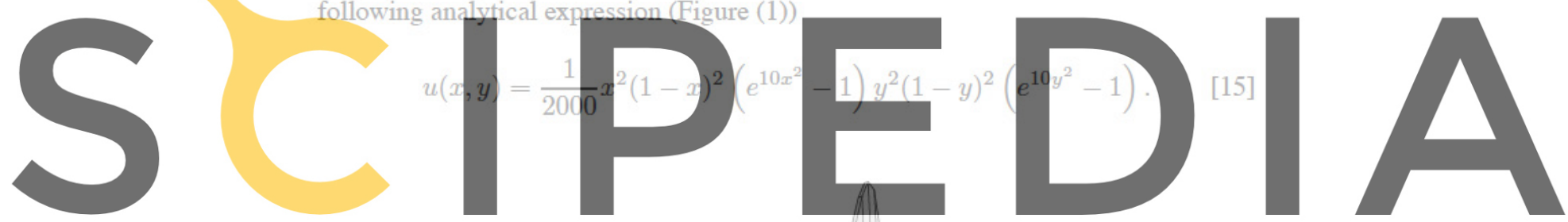

Register for free at https//www.scipedia.com to download the version without the watermark

Figure 1. Example 1: exact solution

Two different non-uniform meshes have been considered (Figure (2)). The reference space is obtained dividing each element of the mesh into $4^{2}$ new elements.

\begin{tabular}{|cc|cccc|}
\hline ndof & $\left\|e_{\text {ref }}\right\|$ & $\rho\left(\varepsilon^{+}\left(e_{\mathrm{u}}\right)\right)$ & $\rho\left(\varepsilon^{+}\left(\eta_{u}\right)\right)$ & $\rho\left(\varepsilon^{-}\left(e_{\mathrm{w}}^{\mathrm{G}}\right)\right)$ & $\rho\left(\varepsilon^{-}\left(\eta_{\mathrm{w}}^{\mathrm{G}}\right)\right)$ \\
\hline \hline 240 & .2447 & .0123 & .3174 & .0066 & .0259 \\
1081 & .1164 & .0024 & .2727 & .0016 & .0292 \\
\hline
\end{tabular}

Table 1. Estimates obtained in example 1 

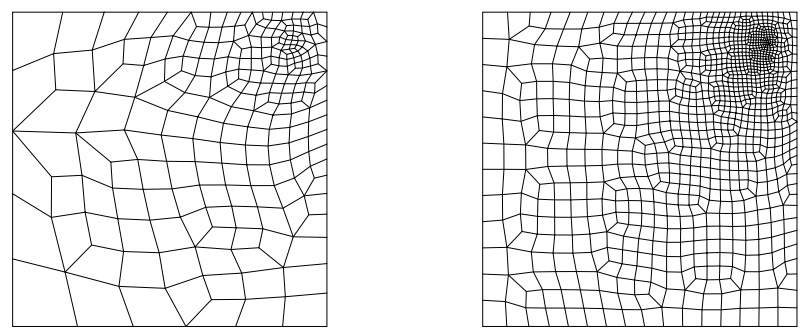

Figure 2. Example 1: initial meshes for example 1

Paradoxically, in this example, the lower bounds provide positive values of the parameter $\rho$. This is because $\varepsilon^{-}\left(e_{\mathrm{w}}^{\mathrm{G}}\right)$ and $\varepsilon^{-}\left(\eta_{\mathrm{w}}^{\mathrm{G}}\right)$ are lower than the exact error but not necessarily lower than the reference error which is used to compute $\rho$. A similar phenomenon occurs for $\varepsilon^{+}\left(e_{\mathrm{u}}\right)$. The estimate $\varepsilon^{+}\left(e_{\mathrm{u}}\right)$ is larger than the reference error but not necessarily larger than the exact error. Then, even if $\left\|e_{\text {ref }}\right\|^{2} \leq \varepsilon^{+}$and $\varepsilon^{-} \leq\|e\|^{2}$ stands, it may happen, see table, that $\varepsilon^{+}\left(e_{\mathrm{u}}\right)<\varepsilon^{-}\left(\eta_{\mathrm{W}}^{\mathrm{G}}\right)$. This can be avoided projecting the continuous functions $e_{\mathrm{W}}^{\mathrm{G}}$ and $\eta_{\mathrm{W}}^{\mathrm{G}}$ into $V^{\text {ref }}$ by a simple nodal interpolation. In this case, $\varepsilon^{-}\left(e_{\mathrm{w}}^{\mathrm{G}}\right)$ and $\varepsilon^{-}\left(\eta_{\mathrm{w}}^{\mathrm{G}}\right)$ are lower bounds for the squared

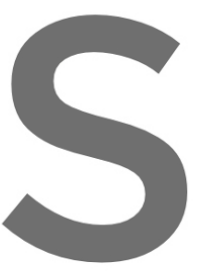

energy norm of the reference error $\left\|e_{\text {ref }}\right\|^{2}$, and thus $\varepsilon^{-}$

The local effectivity indexes associated with $e_{\mathrm{u}}$ are analyzed in the histogtam in Figure (3), which shows the occurrences of the local effectivity indexes. Only elements with a significant contribution to the that the corre of elements) onding 1 al error is lower thar concentrated around $100 \%$ and therefore the local behavior of the estimate is good.

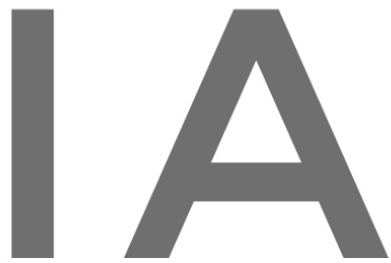

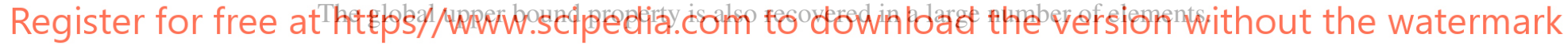

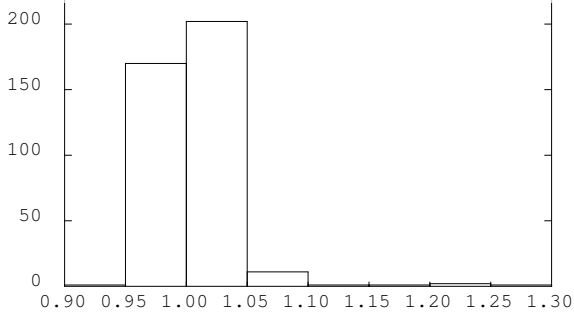

Figure 3. Effectivity index histogram of the estimate $\varepsilon^{+}\left(e_{\mathrm{u}}\right)$ for the mesh with 1081 ndof

In Figure (4) the spatial distributions of the estimate $e_{\mathrm{u}}$ and reference error are shown. Note that the estimate performs well and the plots are practically identical. 

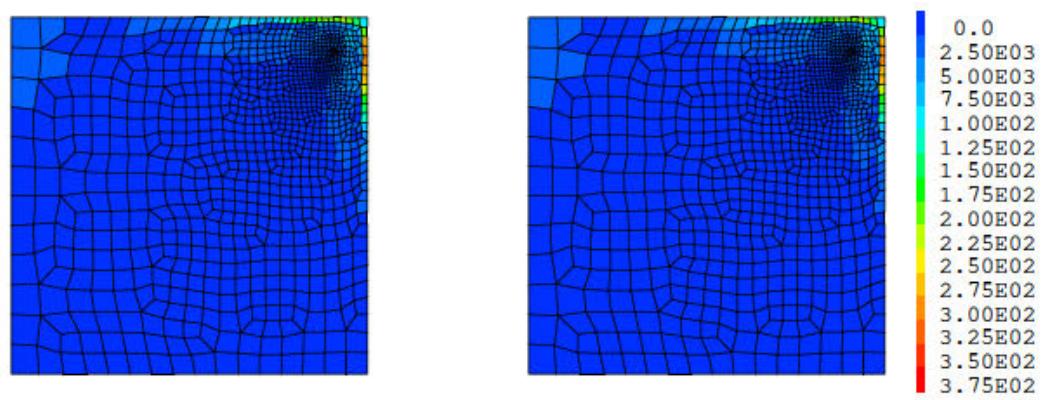

Figure 4. Example 1: spacial distribution of the reference error (left) and the estimated error $\varepsilon^{+}\left(e_{\mathrm{u}}\right)$ (right) for the mesh with 1081 ndof

\subsection{Example 2: plane mechanical problem}

In this section the behavior of the estimate $\varepsilon^{+}\left(e_{\mathrm{u}}\right)$ introduced above and the associated upper and lower bounds is analyzed in a plane-stress linear elasticity problem. The domain is a one-fourth of a symmetrically loaded plate with two rectangular holes.

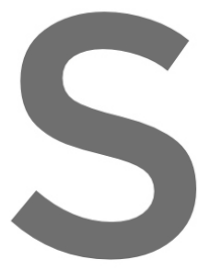
as shown in the symmetry tions on the nor and components respectively
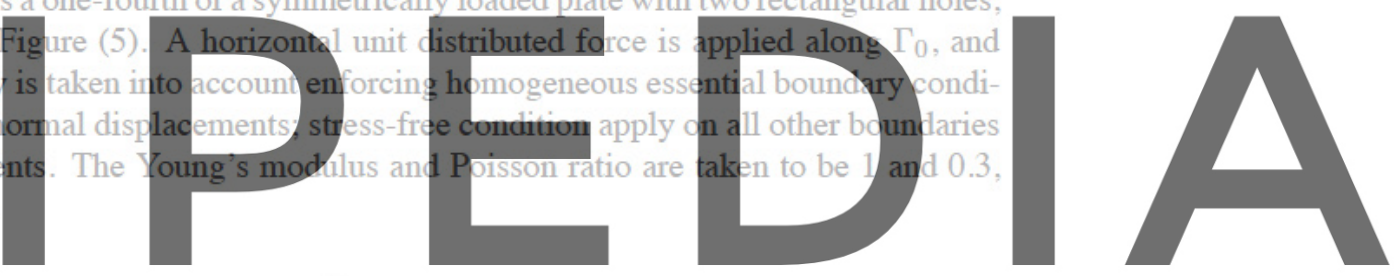

Register for free at https//www.scipedia.com to download the version without the watermark
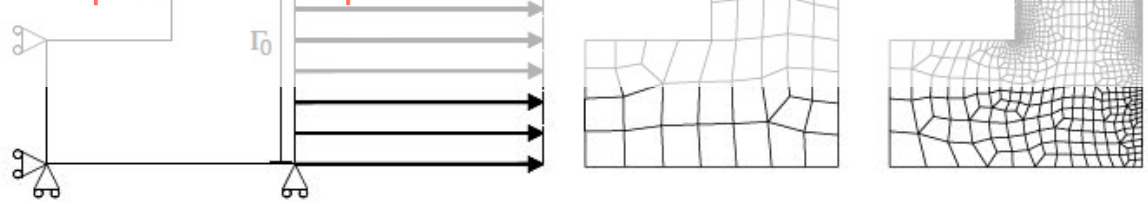

Figure 5. Example 2: model problem and initial meshes with 70 nodes (center) and 1294 nodal points (right)

The considered meshes are shown in Figure (5). For the coarse mesh (70 nodes), the reference mesh is obtained dividing each element into $8^{2}$ new ones, while for the refined one (1 294 nodes) each element is divided into $4^{2}$ new ones.

The results concerning the assessment of the energy norm of the reference error are shown in table 2. In mechanical problems the obtained effectivities for $\varepsilon^{+}\left(e_{\mathrm{u}}\right)$ are not as good as for the previous example. 


\begin{tabular}{|c|cc|cc|}
\hline nodes & $\left\|e_{\text {ref }}\right\|$ & $\frac{\left\|e_{\text {ref }}\right\|}{\left\|u_{h}\right\|}$ & $\rho\left(\varepsilon^{+}\left(e_{\mathrm{u}}\right)\right)$ & $\rho\left(\varepsilon^{-}\left(e_{\mathrm{w}}^{\mathrm{G}}\right)\right)$ \\
\hline 70 & .15434 & .13561 & .21822 & -.42410 \\
1294 & .0354100 & .0308283 & .16310 & -.62755 \\
\hline
\end{tabular}

Table 2. Example 2: upper and lower bounds for $\left\|e_{\text {ref }}\right\|$

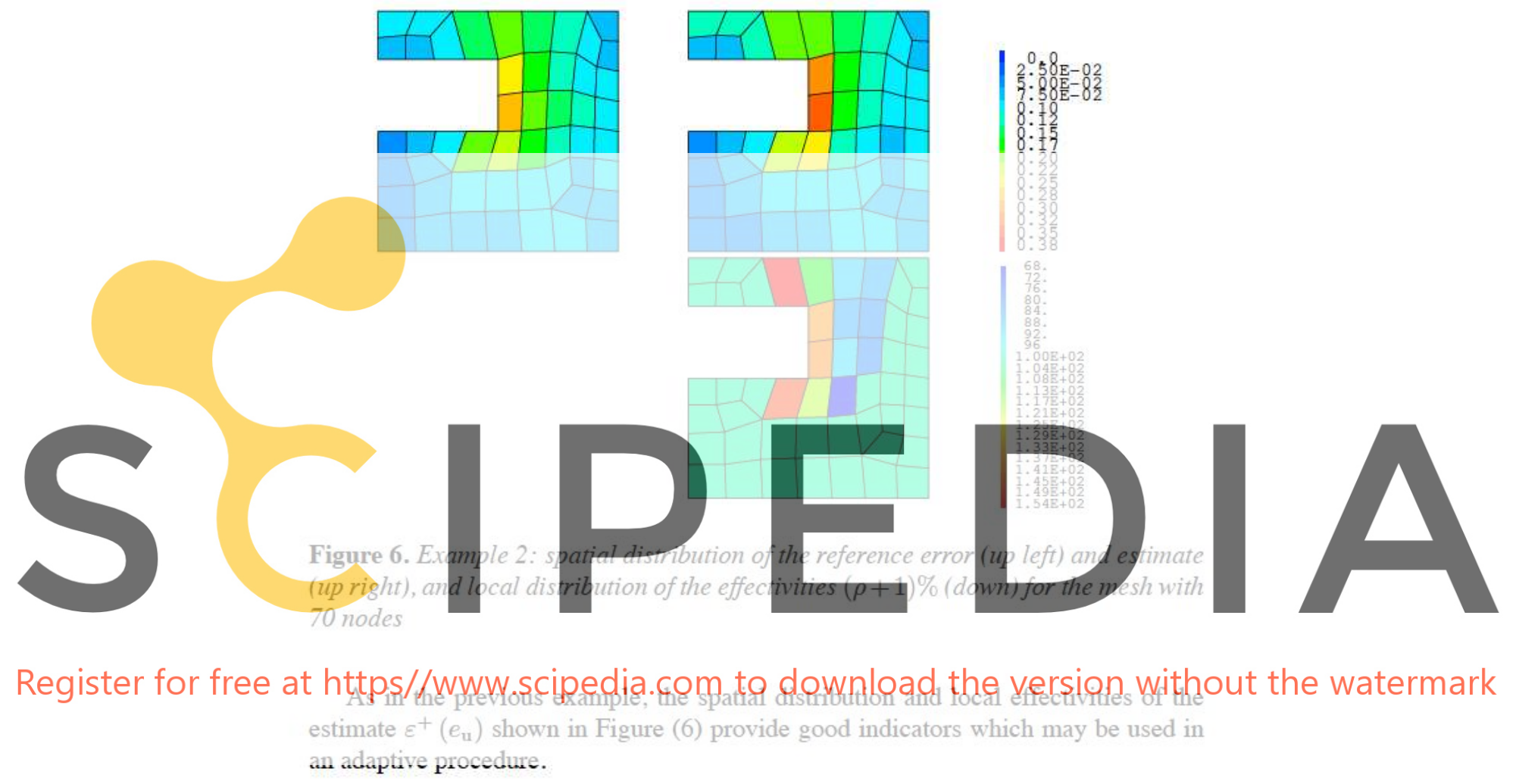

\section{Concluding remarks}

A general strategy to estimate the error in finite element computations has been introduced yielding both upper and lower bounds for the error. The main features of this approach are the following:

- The upper bound is obtained using a residual estimator based on solving local problems on stars (local subdomains). The local problems do not require to compute and equilibrate fluxes. The estimated error function is discontinuous across the element edges.

- A lower bound is obtained by postprocessing the approximation of the error function computed for the upper bound estimate. The error function is smoothed out 
to obtain a continuous function. Then, the error estimate in energy norm is computed in a straightforward manner.

The motivation for obtaining simultaneous upper and lower bounds of the energy norm of the error arises from the necessity of estimating the error in quantities of interest, for goal oriented adaptivity. In this context, the assessment of the energy norm of the error is required for both the primal problem (the original one) and a dual problem related with the selected quantity of interest. In order to obtain accurate bounds of the error in the quantity of interest both upper and lower bounds of the energy norm of the error are required. The present approach is a simple and efficient tool for this purpose.

Compared to other approaches based in the same idea [MAC 00, CAR 99, MOR 03 PRU 02], the strategy introduced here shows a better behavior in the numerical tests, yielding sharper error estimates. Although this is demonstrated in all the studied examples (both mechanical and thermal, 2D and 3D...) a further analysis is required to obtain a general proof of this property.

\section{References}
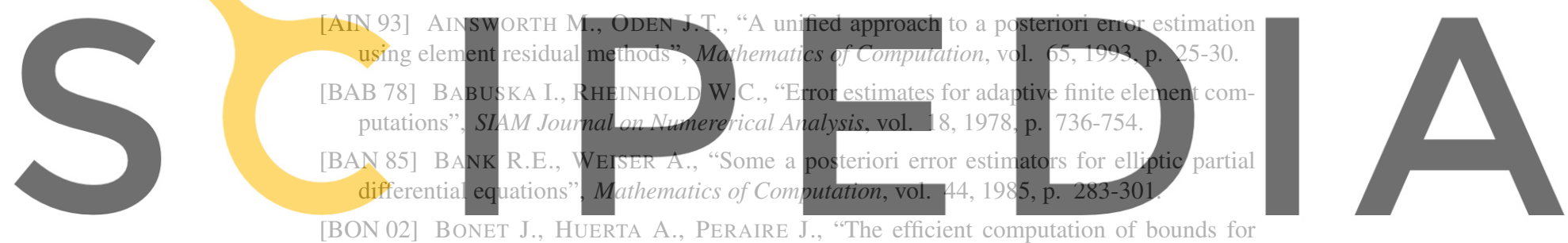

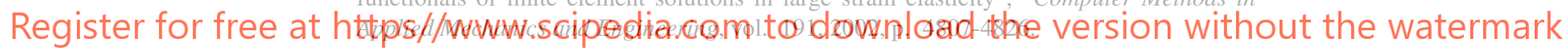

[CAR 99] CARTENSEN C., FUnKEN S.A., "Fully reliable localised error control in FEM", SIAM Journal on Scientific Computing, vol. 21, num. 4, 1999/2000, p. 1465-1484.

[DIE 03] DíEz P., PARÉs N., Huerta A., "Recovering lower bounds of the error postprocessing implicit residual a posteriori error estimates", International Journal for Numerical Methods in Engineering, vol. 56, 2003, p. 1465-1488.

[HUE 00] Huerta A., DíEz P., "Error Estimation Including Pollution Assessment for Nonlinear Finite Element Analysis", Computer Methods in Applied Mechanics and Engineering, vol. 181, 2000, p. 21-41.

[MAC 00] Machiels L., Maday Y., Patera A.T., "A "Flux-Free” Nodal Neumann Subproblem Approach to Output Bounds for Partial Differential Equations", C. R. Acad. Sci. Paris, Série I, vol. 330, num. 3, 2000, p. 249-254.

[MOR 03] Morin P., Nochetto R., Siebert K., "Local problems on stars: a posteriori error estimators, convergence and performance", Mathematics of Computation, vol. 72, 2003, p. 1067-1097. 
[PAR 97] Paraschivoiu M., Peraire J., PATera A.T., “A posteriori finite element bounds for linear-functional outputs of elliptic partial differential equations", Computer Methods in Applied Mechanics and Engineering, vol. 150, 1997, p. 289-312.

[PRU 99] PRUdhomme S., OdEn J.T., "On Goal-Oriented Error Estimation for Elliptic Problems: Application to the Control of Pointwise Errors", Computer Methods in Applied Mechanics and Engineering, vol. 176, 1999, p. 313-331.

[PRU 02] Prudhomme S., Nobile F., Chamoin L., Oden J.T., "Analysis of a subdomainbased error estimator for finite element approximations of elliptic problems", internal report, num. 34, 2002, TICAM. 